\title{
Tuberculous Infection around Shunt Tube-An Unusual Presentation with Review of Literature
}

\author{
Sanjay Kumar ${ }^{1 *}$, Monika Gupta ${ }^{1}$, Sonia Hasija ${ }^{1}$, Ishwar Singh ${ }^{2}$, Sant Prakash Kataria ${ }^{1}$ and Rajeev Sen ${ }^{1}$ \\ ${ }^{1}$ Department of Pathology, PGIMS Rohtak, Haryana, India \\ ${ }^{2}$ Department of Neurosurgery, PGIMS Rohtak, Haryana, India
}

\begin{abstract}
Ventriculoperitoneal shunt is one of the most frequently performed procedures by the neurosurgeons in clinical practice. This simple device results in great improvement in neurological functions and survival of patients with hydrocephalous. Infections associated with the shunt placement vary between 2 to $27 \%$. Central nervous system infection is a cause of significant morbidity and mortality causing malfunction and chronic ill health and a few cases may even turn fatal. They are mainly caused by coagulase negative staphylococcus and rarely by other bacterias like streptococci, enterococci, Gram negative bacilli, fungus etc. We encountered a very rare case in which a 12 year-old female child developed an infection of subcutaneous tissue around the shunt tube that was consistent with M. tuberculosis.
\end{abstract}

Keywords: Ventriculoperitoneal shunt; Tuberculosis; Infection

\section{Introduction}

Ventriculoperitoneal (VP) shunt is one of the most frequently performed procedures in the neurosurgical practice [1]. This simple device results in great improvement in neurological functions and survival of patients with hydrocephalous, at the same time is associated with complications especially infections and malfunctioning. Infections are mainly caused by Coagulase Negative Staphylococcus (CONS) and rarely by other bacterias like streptococci, enterococci, Gram negative bacilli, fungus etc. [2]. Rarely these can also be caused by atypical systemic infections or atypical shunt infections by the mycobacteria group. There is occasional case report in the English literature of shunt tube infection caused by Mycobacterium tuberculosis.

\section{A Case Report}

A 12 year female child suffering from cerebral palsy with hydrocephalous underwent a ventriculoperitoneal shunt placement in the neurosurgery department. Her post-operative recovery was uneventful. However, 20 months later, she presented with history of high grade fever associated with headache and vomiting for last 3 days. There was no associated history of loss of appetite, weight loss or cough with sputum or any other significant medical history. On examination she was febrile and her shunt chamber was collapsed. Mild erythema, swelling and induration along the line of subcutaneous tunnel were noted. On pressing the subcutaneous tunnel the thick creamy material oozed out which was send for culture and sensitivity. Neurologically, she had spastic paraparesis with mental retardation (IQ-46). Her fundus examination was normal and there were no meningeal signs. Other systemic examinations were within normal limits. CT scan head was performed which revealed compensated hydrocephalous. Patient was subjected for investigations to look up the cause of infection. Under local anaesthesia the shunt tube was removed and sent for culture and sensitivity. Local debridement of subcutaneous tissue around shunt tube was performed and material was sent for histopathological examination. CSF examination revealed total leucocyte count of 40 cells/cmm and differential leucocyte count of lymphocytes (55\%) and neutrophils (45\%). Biochemical examination of CSF showed protein $65 \mathrm{mg} \%$ and sugar $40 \mathrm{mg} \%$ whereas corresponding blood sugar was 92 $\mathrm{mg} \%$. Peripheral blood smear did not reveal any abnormality. With a presumptive diagnosis of shunt infection patient was empirically treated with a combination of intravenous antibiotics. Even after seven days of the treatment, no improvement was noted in the patient's condition. She had persistent high grade fever. No bacteria or tubercular baciili seen on Gram staining or Acid-Fast Bacilli (AFB) staining respectively (Figures 1 and 2). Aerobic cultures were also negative for any microbial growth.

Histopathological examination of subcutaneous tissue showed multiple epithelioid cell granulomas with caseous necrosis and Langerhans giant cells. ZN stain for AFB was positive. A diagnosis of

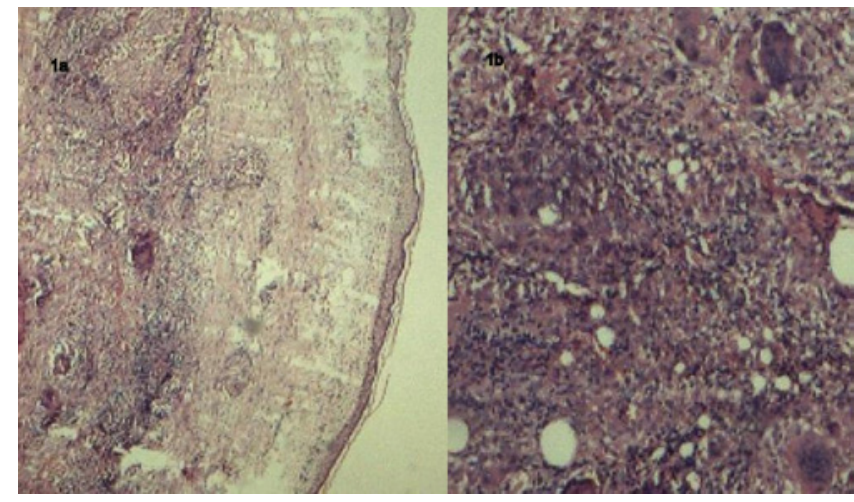

Figure 1: Photograph showing skin covered soft tissue piece with presence of epitheliod cell granuloma with Langerhans giant cells in the subepithelium and dermis. (H\&E 40X) \& numerous giant cells in the subepithelium and dermis. (H\&E 200X).

*Corresponding author: Dr. Sanjay Kumar, 419J, Medical Enclave, PGIMS Rohtak-124001(Haryana), India, Tel: +911262211515; Fax: 911262211308; E-mail: drsanjayk@yahoo.co.uk

Received March 20, 2013; Accepted May 21, 2013; Published May 25, 2013

Citation: Kumar S, Gupta M, Hasija S, Singh I, Kataria SP, et al. (2013) Tuberculous Infection around Shunt Tube-An Unusual Presentation with Review of Literature. Trop Med Surg 1: 123. doi:10.4172/2329-9088.1000123

Copyright: (C) 2013 Kumar S, et al. This is an open-access article distributed under the terms of the Creative Commons Attribution License, which permits unrestricted use, distribution, and reproduction in any medium, provided the original author and source are credited. 


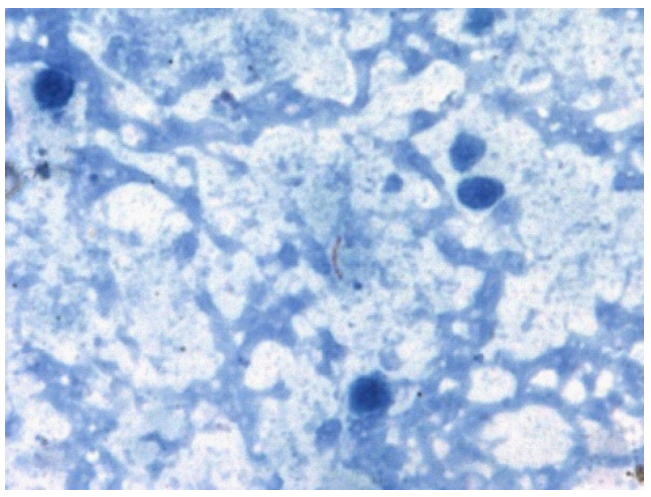

Figure 2: Photograph showing acid fast tubercle bacilli ( ZN stain 1000x).

tuberculous infection of subcutaneous tunnel around shunt was made and Anti-Tubercular Treatment (ATT) with isoniazid, rifampicin, pyrazinamide and ethambutol was started. The patient was negative for finding of primary focus as thereafter investigation. Detailed family history was taken but no history of tuberculosis could be found in the family. Since the patient has compensated hydrocephalous, shunt replacement was not required. Anti-tubercular treatment was given for nine months. She responded very well to ATT treatment with significant improvement in neurological symptoms and on regular follow up till now.

\section{Discussion}

Infection associated with VP shunt placement varies between 2 to $27 \%$ and are associated with significant consequences [1-3]. It ranges from incidently identified and clinically aymptomatic infection to life threatening sepsis which can be fatal to life. The most common bacteria affecting shunts are staphylococcus species followed by streptococci, enterococci, Gram negative bacilli and yeasts. Atypical systemic and shunt infections are also known to occur in such cases [1,2]. The shunt apparatus has proved to promote and perpetuate bacterial growth by protecting bacteria, and thus making them inaccessible to either host defenses or bactericidal drugs [4].

Tuberculosis is a common disease in the developing countries of the world like India. Mycobacterium tuberculosis infects human being via aerosol infection. It can involve any organ or system in the body. Pulmonary tuberculosis is the most common form of presentation, whereas Extrapulmonary Tuberculosis (EPTB) is uncommon but significantly important clinical problem and are more difficult to diagnose compared to their pulmonary counterpart [5]. Several strains of Mycobacteria can produce cutaneous manifestations but $M$. tuberculosis causing post-operative surgical wound infections are uncommon and extremely rare [6]. The immunosuppressive effects of major surgery have also been postulated as a cause for reactivation of tuberculosis $[7,8]$. Tissues seeded with $M$. tuberculosis during primary infection can reactivate many years later by the disruption of local, quiescent granuloma. Spread of the infection occurs by one of the three ways, from exogenous source (inoculation tuberculosis), from endogenous source (secondary tuberculosis) or by local reactivation from hematogenous source. In majority of cases, it is caused by reactivation of dormant tuberculous focus present prior to or during surgical procedure [2].

As shunt infection by M. tuberculosis is very rare, in our case also we treated the patient with a combination of broad spectrum antibiotics considering the surgical site bacterial infection. Local debridement of subcutaneous tissue around shunt tubes was done twice but there was no improvement in the condition of the patient. The diagnosis of tuberculosis was confirmed only after histopathological examination of the debrided tissue. There was no sign of any active focus of tuberculosis in our patient but the cause considered could be either reactivation of an old tubercular foci or use of contaminated materials. Literature also suggests that as long as the VP shunt, a foreign body, is left in situ, there is a high risk of being infected [9].

The most accepted method to diagnose the shunt infection is exteriorization of the shunt and culture of the shunt tube [4]. However, conventional methods were not helpful in this case as exteriorization was not required because patient has compensated hydrocephalous and shunt was non- functioning and culture of the material around shunt in this case was not informative. Diagnosis was made on histopathological examination of the tissue submitted. However as there was no growth on culture, the identification of mycobacterial species was not possible but patient responded well to the standard four-drug antitubercular regimen indicating the probability of $M$. tuberculosis infection.

However, tuberculosis is a major public health problem in developing countries of the world like India. Also there is an increase in incidence of drug resistant tuberculosis which are difficult to treat and contribute to increased mortality. This case report also emphasizes importance of subjecting whatever material is obtained from shunt site for culture and histopathology so that the infective organism like mycobacteria and fungus etc. can be diagnosed and managed.

\section{Conclusion}

Surgical wound infection by $M$. tuberculosis is a rare occurrence still they should not be overlooked in developing and third world countries due to nonspecific clinical manifestations. The diagnosis can be missed unless there is strong clinical suspicion. Because of high frequency and significant morbidity of shunt infection considerable attention is necessary for prevention.

\section{References}

1. Baradkar VP, Mathur M, Sonavane A, Kumar S (2009) Candidal infections of ventriculoperitoneal shunts. J Pediatr Neurosci 4: 73-75.

2. Sarguna P, Lakshmi V (2006) Ventriculoperitoneal shunt infections. Indian J Med Microbiol 24: 52-54.

3. Bokhary MA, Kamal H (2008) Ventriculo-peritoneal shunt infections in infants and children. Libyan J Med 3: 20-22.

4. Kumar R, Mahapatra AK (2007) Unusual manifestations of infection following shunt: An interesting case summary. J Pediatr Neurosci 2: 31-32.

5. Salam MA, Asafudullah SM, Huda MN, Akhter N, Mubinul-Islam ABMM (2011) Surgical site infection by mycobacterium tuberculosis following caesarian section. Pak J Med Sci 27: 945-947.

6. Pattnaik SP, Varma R, Indap S (2012) A rare case of post abdomina hysterectomy scar tuberculous sinus tract formation. Bombay Hospital J 54 : 322-323.

7. Kestler M, Reves R, Belknap R (2009) Pacemaker wire infection with Mycobacterium tuberculosis: a case report and literature review. Int J Tuberc Lung Dis 13: 272-274.

8. Weprin BE, Swift DM (2002) Complications of ventricular shunts. Techniques in neurosurgery $7: 224-242$.

9. Cotton MF, Hartzenberg B, Donald PR, Burger PJ (1991) Ventriculoperitoneal shunt infections in children. A 6-year study. S Afr Med J 79: 139-142. 